\title{
Practical pharmacotherapy in child psychiatry: an update
}

\author{
Rachel Brown \& Anthony James
}

\begin{abstract}
SUMMARY
Psychopharmacology is an increasingly important aspect of child and adolescent psychiatry. The evidence base for the psychopharmacological treatment of depression, anxiety, schizophrenia, bipolar disorder, obsessive-compulsive disorder (OCD) and attention-deficit hyperactivity disorder (ADHD) is increasing. This article presents a synthesis of the information from randomised controlled trials, meta-analyses and the UK NICE guidelines as an aid to practical psychopharmacology.
\end{abstract}

\section{LEARNING OBJECTIVES}

- Describe what current guidelines recommend for the pharmacological management of psychosis, depression, bipolar disorder, OCD, anxiety disorder and ADHD in children and adolescents

- Be aware of the adverse effects of psychotropic medicines, how this may affect treatment choice and what monitoring is required

- Appreciate the limitations to the evidence base supporting the use of psychotropic medicines in this age group

\section{DECLARATION OF INTEREST}

R.B. has received honoraria from Shire Pharmaceuticals Ltd for advisory board and consultancy work

Pharmacotherapy is an increasingly important tool in child and adolescent psychiatry. In many highincome countries there has been a large increase in the prescription of psychotropic medication for children and adolescents, often 'off-label' (Box 1) and often for conditions where evidence is lacking.

In this review, we refer primarily to randomised controlled trials (RCTs), guidelines from the National Institute for Health and Care Excellence (NICE) and Cochrane reviews. A number of previous reviews of drug treatments for child psychiatric disorders have included open trials, but this leaves the potential for biases. However, a third of registered trials may remain unpublished (Ross 2012), which is a serious issue and, indeed, the non-publication of data may alter current recommendations and guidelines. With these caveats in mind, we provide an overview of the following conditions where medication is an important component of treatment in child psychiatry: depression, psychoses, bipolar disorder, obsessivecompulsive disorder (OCD), anxiety disorders and attention-deficit hyperactivity disorder (ADHD).

Although this review is limited to medication, psychological treatments are effective in this age group and NICE guidelines recommend psychological treatments as first line for a number of disorders, including OCD, depression, anxiety disorder, psychosis and bipolar disorder.

\section{Depression}

UK guidance recommends a stepped-care approach, with psychological therapy for all those with mild depression and, in 12- to 18-year-olds, psychological therapy combined with fluoxetine for moderate to severe depression (NICE 2005a). For 5to 11-year-olds fluoxetine is considered only when psychological therapy has been ineffective. Despite numerous studies attempting to determine whether efficacy differences exist between psychological interventions, antidepressant medication and their combination for the treatment of depressive episodes, a Cochrane review concluded that the optimum strategy is not known (Cox 2014). Indeed, combined treatment seems to have an

\section{BOX 1 Off-label and unlicensed medicines}

In the UK, the Medicines and Healthcare products Regulatory Agency (MHRA) grants product licences to medicines when clinical trials have demonstrated safety and efficacy. The licence is usually for a specific indication and group of people - e.g. adults. Using a licensed medicine for a different indication or in an age group not covered by the product licence is referred to as off-label or off-licence use. Unlicensed medicines include those made in the UK by specialist manufacturers (e.g. a liquid formulation of sertraline) or medicines imported from other countries, even if they have been granted a licence in the country of origin. It is accepted practice in the UK that licensed medicines can be used off-label in children and adolescents when no suitable licensed alternative is available or where use is justified by a responsible body of professional opinion.

(Royal College of Psychiatrists 2007)

\section{ARTICLE}

Rachel Brown is a mental health pharmacist. She runs the Medicines Information Service for Oxford Health NHS Foundation Trust, is the clinical lead pharmacist for the Children and Young People's Directorate and works closely with the in-patient team at the Highfield Adolescent Unit at the Warneford Hospital, Oxford. Anthony James is a consultant child and adolescent psychiatrist working with in-patients at the Highfield Adolescent Unit at Warneford Hospital, Oxford and an honorary senior lecturer at the University of Oxford.

Correspondence Rachel Brown, Oxford Health NHS Foundation Trust, Clinical Pharmacy Support Unit, Unit 46, Sandford Lane, Kennington, Oxford OX1 5RW, UK. Email: rachel. brown@oxfordhealth.nhs.uk 
unspecific effect on global functioning and general improvement, rather than on depressive symptoms (Calati 2011).

In 2003, considerable alarm arose following the 'black box' warnings by the US Food and Drug Administration (FDA) and the UK Medicines and Healthcare products Regulatory Agency (MHRA) over the possible association of selective serotonin reuptake inhibitors (SSRIs) with increased suicidal ideation and suicide attempts. Available evidence is inconclusive: a relatively recent metaanalysis found no evidence of increased suicide risk in children and adolescents taking fluoxetine (Gibbons 2012). Although the mechanism of any proposed association between self-harm and SSRI use is not yet clear, a prospective US population healthcare study found that the use of higher than modal antidepressant doses was associated with twice the rate of self-harm in individuals under 25 years of age (Miller 2014). A Cochrane review (Hetrick 2012) found a small positive benefit on depression rating scales and remission rates with newer generation antidepressants (paroxetine, fluoxetine, citalopram, escitalopram, sertraline, venlafaxine and mirtazapine), but with an increased risk $(58 \%)$ of suicide-related outcomes for those on antidepressants compared with placebo (17 trials; $n=3229 ; \mathrm{RR}=1.58 ; 95 \% \mathrm{CI}$ 1.02-2.45). A prospective 24 -week study found a higher overall incidence of suicidal behaviour and/ or ideation on the clinician-rated Columbia-Suicide Severity Rating Scale (C-SSRS) in adolescents treated with escitalopram (14.5\%) compared with placebo (10.9\%) (Findling 2013a). Close monitoring for adverse effects, particularly early in treatment, remains necessary.

Fluoxetine is the most widely studied antidepressant in young people, and plasma levels appear to be similar to those achieved in adults and not influenced by age (Blazquez 2014). Although fluoxetine is the recommended antidepressant for the treatment of depression in children and adolescents in the UK, in practice clinicians prescribe a wider variety of drugs. NICE (2005a) advocates the use of citalopram or sertraline when response to fluoxetine is poor. Venlafaxine and paroxetine should be avoided, as they have the strongest association with suicide risk; tricyclic antidepressants (TCAs) are not useful in treating depression in young children and there is only lowquality marginal evidence to support their use in adolescents (Hazell 2013).

\section{Prescribing of antidepressants}

Starting doses of antidepressants, especially in children, should be low (e.g. fluoxetine
B0X 2 Selective serotonin reuptake inhibitors (e.g. fluoxetine, sertraline, citalopram): adverse effects and management

Nausea - common, particularly at the start of treatment, dose related and usually transient. Minimise by starting with low doses and taking with food. In severe cases it can be alleviated with a short course of anti-emetic.

Anxiety - occasionally may be exacerbated, particularly at the start of treatment, but SSRIs are anxiolytic in the long term. Provide advice and reassurance.

Bleeding - increased risk of bruising, nose bleeds, gastrointestinal bleeding and prolonged bleeding time. Absolute risk is very small and comparable to the relative risk with aspirin or other non-steroidal anti-inflammatory drugs (NSAIDs).

Sexual dysfunction and loss of libido - common, not usually transient.

Insomnia - common, helped by taking the dose in the morning or a dose reduction.

Serotonin syndrome - potentially life-threatening. Initial presentation may include mild symptoms such as tachycardia, shivering, sweating, mydriasis, diarrhoea and tremor, but if overlooked can quickly escalate to life-threatening delirium, neuromuscular rigidity and hyperthermia. Serotonin syndrome is more likely with higher doses or when used in combination with other drugs that increase serotonin levels (e.g. other antidepressants, lithium, triptans, tramadol).

$10 \mathrm{mg} /$ day), and the dose gradually titrated according to response and side-effects (Box 2). For more detailed dosing information we refer readers to the most recent $B N F$ for Children (www.bnf.org) and the manufacturer's Summary of Product Characteristics (www.medicines.org. $\mathrm{uk} / \mathrm{emc}$ ). There is some evidence that continuing antidepressant medication reduces the chance of relapse, and relapse may be lower with the addition of cognitive-behavioural therapy (CBT) (Kennard 2014). It has been suggested that antidepressant treatment continue for up to 6 months after initial remission.

\section{Treatment resistance ${ }^{a}$}

About $40 \%$ of depressed adolescents do not respond to treatment. If treatment resistance occurs, the Texas guideline (Hughes 2007) recommends a change in SSRI, or a change in class of antidepressant and/or augmentation. The Treatment of Resistant Depression in Adolescents (TORDIA) study found that SSRI non-responders whose treatment was augmented with a mood stabiliser (atypical antipsychotic, lithium, divalproex, topiramate) or with CBT 
during the first 12 weeks of treatment were more likely to remit (Emslie 2010). However, while this study and the Treatment for Adolescents With Depression Study (TADS) (March 2009) found the addition of CBT positive, the UK Adolescent Depression Antidepressants and Psychotherapy Trial (ADAPT) study (Goodyer 2008) found no added benefit for CBT over SSRIs alone. In treatment-refractory depression with the risk of suicide, electroconvulsive therapy (ECT) should be considered; however, although evidence is available for adults, no RCTs have been carried out in children and adolescents.

\section{Anxiety disorders}

There is an extensive literature on psychological treatments such as CBT for anxiety disorders (James 2015), and psychological treatments are regarded as first-line treatment. Although no medication currently has MHRA approval for use in anxiety disorders in children and adolescents, there is, nonetheless, a growing literature on drug treatments - mainly SSRIs - for these disorders. A recent review (Mohatt 2014) found evidence for the efficacy of SSRIs in the treatment of a range of anxiety disorders - separation anxiety, social anxiety, generalised anxiety - and comorbid anxiety disorders in children and adolescents. Combination treatment - SSRIs and CBT - have been found to be more effective than either alone.

\section{Psychosis and schizophrenia}

Psychological therapies are recommended as firstline treatment (NICE 2013), particularly in the early transitional stages of psychosis. Antipsychotic medication, however, remains a mainstay of treatment in combination with psychological therapies - CBT and family therapy. Unfortunately, there is a relative lack of good-quality data on which to base treatment recommendations in young people with schizophrenia or psychosis. A Cochrane review (Kumar 2013) found no convincing evidence for a difference in efficacy between first-generation (typical) and secondgeneration (atypical) antipsychotics, but the second-generation drugs are more acceptable as they have fewer symptomatic side-effects in the short term (Kumar 2013). Antipsychotics may be less efficacious in young people than in adults (NICE 2013).

\section{Prescribing of antipsychotics}

Only aripiprazole (15-year-olds and older) and clozapine (16-year-olds and older) are licensed in the UK for patients under 18, but in practice licensing does not seem to affect prescribing choice.
Second-generation antipsychotics are almost universally chosen (aripiprazole, amisulpride, olanzapine, quetiapine and risperidone). Involving the patient in the choice wherever possible is important as adverse effect profiles of secondgeneration antipsychotics differ significantly. There is some evidence that lower doses may be sufficiently effective, but with risperidone it appears that adolescents may respond better to standard doses as opposed to lower doses (Kumar 2013). Nonetheless, it is advisable to start low and to increase slowly to minimise dose-related side-effects and to establish the lowest effective dose, particularly as the burden of side-effects with antipsychotics appears to be greater in young people. Response to antipsychotics can be early, with week-3 improvements having the best predictive power, at least for aripiprazole (Correll 2013a). Unfortunately, discontinuation rates for antipsychotics are substantial - in one study ( $n$ =110), 44.5\% had dropped out at 6 months, 59.1\% at 12 months and $70.9 \%$ at 24 months (Noguera 2013) - a crucial factor when considering drug treatments.

\section{Treatment resistance}

Failed treatment after adequate (in duration and dosage) trials of two antipsychotics is an indication to use clozapine. The initiation of clozapine requires regular full blood counts and close physical monitoring (pulse, temperature and blood pressure) to detect potential serious sideeffects such as neutropenia, agranulocytosis and myocarditis. Plasma clozapine levels associated with a good clinical response in adults are in the range of 0.35 to $0.60 \mathrm{mg} / \mathrm{L}$. The range has not been determined in young people, although data from a therapeutic drug monitoring service supports the use of plasma clozapine levels to guide dosing in much the same way as in adults (Couchman 2013). In a systematic review of studies in children and adolescents (Schneider 2014) an average improvement of $69 \%$ was found in short-term clinical trials, which was sustained during longterm follow-up of up to 9 years. No deaths linked to clozapine treatment were reported. Neutropenia was reported in $6-15 \%$ of cases, but was usually transient; agranulocytosis was rare $(<0.1 \%)$. Seizures occurred in less than 3\%. Fortunately, the overall rate of discontinuation was low (36\%). Despite a higher incidence of adverse effects in children, clozapine appears to be a uniquely beneficial second-line agent for treating children with refractory schizophrenia. Only clozapine, among the antipsychotics, is associated with a reduced longer-term standardised mortality rate, 
which may partly be due to continued monitoring and the reduced risk of suicide. Unfortunately, evidence for effective augmentation of clozapine in refractory cases is not convincing.

\section{Side-effects}

Weight gain is a major concern with secondgeneration antipsychotics, particularly with olanzapine. Metabolic disturbances with altered lipids and blood glucose are frequent. In a large retrospective cohort study of the Tennessee Medicaid programme, children 6 to 17 years of age prescribed second-generation antipsychotics for non-psychotic disorders had a nearly threefold increased risk of type II diabetes ( $\mathrm{HR}=2.89 ; 95 \%$ CI 1.64-5.100) - a risk that increased significantly with increasing cumulative dose $(P<0.03)$ (Bobo 2013). Worryingly, the risk remained elevated for up to 1 year following discontinuation of antipsychotics. Baseline physical tests and frequent monitoring are essential, as is regular weighing. Information about diet and recommendations to increase exercise should be provided, but if these measures fail and the antipsychotic cannot be switched to one with a lower risk, the addition of metformin, an antidiabetic agent, can be beneficial (Correll 2013b).

Antipsychotic-induced hyperprolactinaemia is very common with antipsychotics such as amisulpride and risperidone. Hyperprolactinaemia can cause gynaecomastia, galactorrhoea and sexual dysfunction, as well as hypogonadism, which can lead to a reduced bone mineral density. In a population who have yet to achieve their peak bone mass this could have serious long-term implications. Baseline prolactin measurements should be taken in all young people prescribed prolactin-raising antipsychotics. It is essential to monitor for symptoms of hyperprolactinaemia during treatment. Switching to a different antipsychotic may be necessary. However, when symptoms occur and the antipsychotic cannot be changed, the addition of aripiprazole, which lowers prolactin levels, may be an alternative management strategy. Referral to a paediatric endocrinologist may also be advisable, particularly in prepubertal children and in adolescents with delayed puberty or failure to progress in puberty.

\section{Bipolar affective disorder}

NICE has recently updated its 2006 guideline (NICE 2014), which we summarise here.

\section{Mania}

In almost 9 years, aripiprazole is the only medicine to have been granted a product licence to treat young people with this condition in the UK. Lithium (Liskonum ${ }^{\circledast}$ only) is the only other licensed medicine for treating manic episodes in this country. In practice, lithium is not used acutely as it requires commitment from the patient to the idea of longer-term treatment and regular blood tests. The dose is also usually titrated gradually to reduce the risk of adverse effects, which may cause a delay in therapeutic response. NICE (2014) recommends considering aripiprazole or using an alternative antipsychotic in accordance with guidance for adults and normally limiting drug treatment to a maximum of 12 weeks. A number of published RCTs have shown beneficial effects with the second-generation antipsychotics compared with placebo in those aged between 6 and 18 years: aripiprazole (Findling 2009; Tramontina 2009), olanzapine (Tohen 2007), quetiapine (Pathak 2013) and risperidone (Haas 2009). Evidence is generally of low quality and, unfortunately, no head-to-head studies exist, so choice is guided by side-effects and patient preference.

It is unclear whether valproate, as a mood stabiliser, has the same beneficial effects on mania in young people as it does in adults. Evidence is conflicting for valproic acid in comparison with antipsychotics. A greater reduction in manic symptoms and better response and remission rates were achieved with risperidone compared with valproic acid in two controlled studies (Pavaluri 2010; Geller 2012a), but at the expense of a higher level of side-effects, including weight gain and hyperprolactinaemia. In a small study of 50 adolescent in-patients with mania, valproic acid was as effective as quetiapine (Delbello 2006). NICE recommends that valproate monotherapy should not be included in the first-line management of acute mania and it should not be offered to girls or young women of childbearing potential because of teratogenic effects and the risk of polycystic ovary syndrome (NICE 2014).

\section{Bipolar depression}

NICE (2014) recommends a 3-month psychological intervention as first-line management of bipolar depression. Unfortunately, there is a lack of goodquality data to support specific drug treatment recommendations. In contrast to studies in adults, results with quetiapine have so far been disappointing (Delbello 2009; Findling 2014), and therefore there is little evidence to recommend quetiapine in young people. Limited evidence suggests that the combination of fluoxetine and olanzapine may be beneficial (Eli Lilly \& Company 2013). If a pharmacological intervention needs to be added in moderate or severe bipolar depression, 
NICE recommends following the guidance for adults (use an antipsychotic or mood stabiliser, consider stopping any antidepressant, or consider continuing the antidepressant but adding an antipsychotic), modifying doses and limiting treatment to 12 weeks only (NICE 2014).

\section{Longer-term treatment}

There is some evidence for using lithium, valproate or aripiprazole as longer-term treatments of bipolar disorder in children and young people (Findling 2005, 2012, 2013b). However, each study had its limitations, notably the high attrition rates with aripiprazole. There is a lack of good-quality evidence to help formulate the optimal long-term management strategy. Although in this age group evidence is scant (Findling 2013c), lithium is seen as a good mood stabiliser, with a very positive and early response in a third of patients (Malhi 2014). Lithium is also protective against suicide, and is thought to be neuroprotective (Rybakowski 2014). With its narrow therapeutic index and potential adverse effects, lithium requires close monitoring, including regular plasma levels, renal function (estimated glomerular filtration rate, eGFR), thyroid function and calcium levels. Curiously, UK guidance currently recommends not routinely continuing antipsychotic treatment in this age group for more than 12 weeks, and using psychological interventions in the longer term (NICE 2014).

\section{Obsessive-compulsive disorder}

UK guidance issued by NICE in 2005 uses a stepped-care model for treatment of OCD. For initial management in children and young people, guided self-help or CBT including exposure response prevention (ERP) with school and family/ carer involvement are recommended. SSRIs are recommended as a first-line drug treatment (NICE 2005b). A recent large meta-analysis of 14 RCTs (Ivarsson 2015) found SSRI treatments for paediatric OCD to be moderately effective, with an effect size (Hedge's $g$ ) of 0.48 or a mean difference of 3.9 points on the Children's Yale-Brown Obsessive Compulsive Scale (CY-BOCS). SSRIs did not vary in their efficacy, but clomipramine, a tricyclic serotonin reuptake inhibitor, may be more effective. Clomipramine is associated with more frequent adverse events and possible cardiac arrhythmias, and hence it is not a first-line choice.

Importantly, the review by Ivarsson et al showed that the effects of CBT were robust and exceeded those of SSRIs; furthermore, combination treatment was not superior to CBT alone. Indeed, according to the authors there were no data showing at which level of severity combination treatment was preferable (Ivarsson 2015). However, previous evidence from the Pediatric OCD Treatment Study (POTS) did find the addition of $\mathrm{CBT}$ to existing maintenance medication to be beneficial, with large effect sizes (POTS Team 2004; Franklin 2011).

The findings of the Ivarsson et al review clearly do not support the recommendation of the American Academy of Child and Adolescent Psychiatry (AACAP) (Geller 2012b) that moderate to severe OCD should be treated with combination therapy from the start. Nevertheless, it seems clear that SSRI non-responders or partial responders should be treated with CBT plus a continued SSRI, although this is based on just one study. It also seems sensible that, according to NICE, SSRIs should be added when the patient prefers drug treatment, or when CBT is not effective, not accepted or not adequately adhered to.

Only two SSRIs have UK product licences in this age group (sertraline for age 6 years and older and fluvoxamine for age 8 years and older). There appears to be relatively more efficacy data in favour of sertraline in both the short and longer term. In the USA, fluoxetine is licensed for OCD but in the UK, NICE recommends its use in OCD only where there is a comorbid diagnosis of depression (NICE 2005b). High doses are often required and the full effect of a particular dose may not become apparent for up to 12 weeks (NICE 2005b).

When response has been poor, AACAP suggests the combined use of an SSRI with clomipramine (Geller 2012b). This is a risky strategy and should be reserved for only the most difficult cases, owing to the increased risk of serotonergic adverse effects and the potential for drug interactions resulting in raised clomipramine levels and toxic cardiac effects.

A more commonly used approach is augmentation with a low-dose antipsychotic, often with risperidone. However, literature supporting the use of antipsychotic augmentation of SSRIs is limited and there are no double-blind RCTs in children or young people. The support given by NICE for this strategy appears to have been extrapolated from studies in adults. Additional monitoring for antipsychotic side-effects is necessary (see earlier).

\section{Attention-deficit hyperactivity disorder}

UK and US recommendations for treating ADHD differ. In the UK, a stronger emphasis is placed on group-based training and education programmes and psychological interventions, and medication is not recommended in preschool children (NICE 
2008). In the USA, there are two guidelines (also differing slightly); AACAP recommends stimulant medication, behavioural therapy or a combination as first line, making no distinction by age (Geller 2012b), whereas and the American Academy of Pediatrics (AAP) recommends behavioural therapy first line in preschoolers, with medication used if the clinician feels it is appropriate (Subcommittee on Attention-Deficit/Hyperactivity Disorder 2011). For school-aged children in the UK with moderate or severe ADHD that has not responded to nondrug management, medication is recommended. In most cases this is the stimulant methylphenidate (NICE 2008), which has a substantial evidence base. This is also the recommended first-line stimulant in European guidelines (Taylor 2004). The non-stimulant atomoxetine, a selective noradrenaline reuptake inhibitor (SNRI), is an alternative when tics, Tourette syndrome, anxiety disorder, stimulant misuse or risk of diversion are present, or where methylphenidate is ineffective or not tolerated (NICE 2008).

The precise mode of action of drugs used to treat ADHD is unknown, but enhancement of dopaminergic and noradrenergic neurotransmission in the prefrontal cortex is probably critical to therapeutic efficacy (Bolea-Alamañac 2014). Methylphenidate blocks reuptake of dopamine and increases noradrenaline levels. Atomoxetine and a drug available in the USA, guanfacine extended release, are both highly selective noradrenergic drugs (Bolea-Alamañac 2014). Guanfacine is an alpha adrenergic receptor agonist pharmacologically similar to clonidine, but with a 15 to 20 times higher affinity for the alpha-2A receptor subtype compared with the alpha-2B and alpha-2C subtypes. The most common side-effects of guanfacine are somnolence, sedation and fatigue. These side-effects may be viewed as beneficial for some individuals, as many patients with ADHD have sleep difficulties that stimulants may worsen. Other side-effects can include hypotension, bradycardia and weight gain. Consequently, careful monitoring of weight, pulse and blood pressure is necessary, in keeping with other ADHD treatments. Guanfacine may not be available in the UK until 2016. Its efficacy is demonstrated in a meta-analysis of placebo controlled trials (Ruggiero 2014), which showed response rates similar to those seen with methylphenidate (Bolea-Alamañac 2014), although it may be less effective at treating the inattentive subtype of ADHD (Bolea-Alamañac 2014).

The stimulant dexamfetamine is included in UK guidance as a second- or third-line treatment option, but it requires multiple daily dosing. In practice it is not often prescribed, despite good evidence of efficacy (Bolea-Alamañac 2014). Lisdexamfetamine, a long-acting prodrug of dexamfetamine used in the USA, has recently been launched in the UK as a second-line treatment. It is pharmacologically inactive but, after absorption from the gastrointestinal tract, it is hydrolysed primarily by red blood cells to active dexamfetamine. This results in a prolonged release effect over the whole day with only oncedaily administration. Placebo controlled studies demonstrate efficacy over 4-9 weeks (Coghill 2014a). The only head-to-head study showed better efficacy in comparison with atomoxetine (Dittman 2013). Data from controlled long-term trials are limited, although a double-blind 6 -week randomised withdrawal study $(n=157)$ found that a significantly higher proportion of patients who met criteria for treatment failure had been assigned to placebo than to the group that had continued with lisdexamfetamine (Coghill 2014b). Lisdexamfetamine shares a similar side-effect profile and requirements for monitoring as other stimulant medication (Box 3), with possible lower misuse potential.

BOX 3 Stimulants (e.g. methylphenidate, lisdexamfetamine, dexamfetamine): adverse effects and management

Nervousness and insomnia - very common on initiation, managed by adjusting the dose.

Abdominal pain, nausea and vomiting - common on initiation, usually transient and can be helped by taking with food.

Headache - very common, treated with simple analgesia such as paracetamol.

Increases in heart rate and blood pressure - take a baseline, measure after each dose change, at 3 months and then 6-monthly. If there is sustained resting tachycardia, a significant increase in pulse (e.g. $20 \mathrm{bpm}$ ) or arrhythmia, or if blood pressure is raised above normal or significantly increased: reduce dose or stop and refer to a cardiologist.

Decreased appetite, reduced weight gain and slight growth retardation - plot height and weight on growth charts and review regularly.

Development of new or worsening of pre-existing psychiatric symptoms - monitor at every dose change and at least 6-monthly.

Tics - can be caused by or worsened by stimulants: review risks and benefits of continuing treatment.

Seizures - if exacerbated in a child with epilepsy, or $d e$ novo seizures emerge, discontinue immediately. 
Once-daily preparations are usually preferred as they improve adherence and avoid the need to take the medication at school. Different methylphenidate preparations vary in their release characteristics and time to be taken in relation to food, and are not interchangeable.

Despite newer options, ADHD may remain refractory or individuals may not tolerate conventional treatments. Consequently, trials of other drugs may be necessary, but the evidence base is sparse. Other treatment options include highdose stimulants and clonidine (Bolea-Alamañac 2014). Limited evidence supports the use of tricyclic antidepressants (Otasowie 2014), but cardiac safety is a concern and European guidelines (Taylor 2004) suggest restricting use to specialist practice. Bupropion has some efficacy in ADHD, but there is a risk of seizures. As bupropion is a cytochrome P450 2D6 (CYP2D6) inhibitor, care is required if used with other medicines metabolised by this enzyme, such as antidepressants (e.g. fluoxetine) and antipsychotics (e.g. risperidone), because the risk of adverse effects may be increased if doses are not adjusted. Modafinil, a central stimulant licensed in the UK only as a wake-promoting agent in narcolepsy, may have some efficacy in ADHD, however the European Medicines Agency's Committee for Medicinal Products for Human Use (2010) concluded that the benefits of modafinil outweighed the risks only in narcolepsy. A Cochrane review found little evidence that polyunsaturated fatty acids provide any benefit in ADHD in young people (Gillies 2012).

\section{Discussion}

It appears that the evidence base for pharmacology in child psychiatry, although growing, is still relatively limited. Medicines are effective, but findings from research trials show the effects sizes, with the notable exception of stimulants for $\mathrm{ADHD}$, to be low to medium. Furthermore, medicines can have considerable side-effects; indeed, the weight gain with second-generation antipsychotics has been described as a potential public health crisis (Reeves 2013). In our experience, despite these reservations, clinical practice, especially with the more ill patients, shows that medicines are a vital component of child psychiatry. It is accepted practice in the UK that licensed medicines can be used offlabel in this age group when no suitable licensed alternative is available or where use is justified by a responsible body of professional opinion (Royal College of Psychiatrists 2007). Nevertheless, there must be caution against the more widespread use of antipsychotics and medication in general, especially in the absence of good evidence.
It is clear from this review that there is a pressing need for more research in this age group, with an emphasis on adherence, longerterm studies, combination treatments and the treatment of resistant conditions. Although there are legitimate concerns about industryfunded research, psychopharmacology research is in a perilous situation, particularly with drug companies withdrawing from this area.

\section{References}

Blazquez A, Mas S, Plana MT, et al (2014) Plasma fluoxetine concentrations and clinical improvement in an adolescent sample diagnosed with major depressive disorder, obsessive-compulsive disorder, or generalized anxiety disorder. Journal of Clinical Psychopharmacology 34: 318-26.

Bobo WV, Cooper WO, Stein CM, et al (2013) Antipsychotics and the risk of type 2 diabetes mellitus in children and youth. JAMA Psychiatry, 70: 1067-75.

Bolea-Alamañac B, Nutt DJ, Adamou M, et al (2014) Evidence-based guidelines for the pharmacological management of attention deficit hyperactivity disorder: update on recommendations from the British Association for Psychopharmacology. Journal of Psychopharmacology, 28: 179-203.

Calati R, Pedrini L, Alighieri S, et al (2011) Is cognitive behavioural therapy an effective complement to antidepressants in adolescents? A meta-analysis. Acta Neuropsychiatrica, 23: 263-271.

Coghill DR, Banashewski T, Lecendreux M, et al (2014a) Efficacy of lisdexamfetamine dimesylate throughout the day in children and adolescents with attention deficit/hyperactivity disorder: results from a randomized, controlled trial. European Child and Adolescent Psychiatry, 23: 61-8.

Coghill DR, Banaschewski T, Lecendreux M, et al (2014b) Maintenance of efficacy of lisdexamfetamine dimesylate in children and adolescents with attention deficit/hyperactivity disorder: randomized withdrawal study design. Journal of the American Academy of Child and Adolescent Psychiatry, 53: 647-57.

Committee for Medicinal Products for Human Use (2010) Modafinil: Summary. EMA

Correll CU, Zhao J, Carson W, et al (2013a) Early antipsychotic response to aripiprazole in adolescents with schizophrenia: predictive value for clinical outcomes. Journal of the American Academy of Child and Adolescent Psychiatry 52: 689-98.

Correll CU, Sikich L, Reeves G, et al (2013b) Metformin for antipsychoticrelated weight gain and metabolic abnormalities: when, for whom, and for how long? American Journal of Psychiatry, 170: 947-52.

Couchman L, Bowskill SVJ, Handley S, et al (2013) Plasma clozapine and norclozapine in relation to prescribed dose and other factors in patients aged <18 years: data from a therapeutic drug monitoring service 19942010. Early Intervention in Psychiatry, 7: 122-30.

Cox GR, Callahan P, Churchill R, et al (2014) Psychological therapies versus antidepressant medication, alone and in combination for depression in children and adolescents. Cochrane Database of Systematic Reviews, 11: CD008324.

Delbello MP, Kowatch RA, Adler CM, et al (2006) A double-blind randomised pilot study comparing quetiapine and divalproex for adolescent mania. Journal of the American Academy of Child and Adolescent Psychiatry, 45: 305-13.

Delbello MP, Chang K, Welge JA, et al (2009) A double-blind, placebocontrolled pilot study of quetiapine for depressed adolescents with bipolar disorder. Bipolar Disorders, 11: 483-93

Dittman RW, Cardo E, Nagy P, et al (2013) Efficacy and safety of lisdexamfetamine dimesylate and atomoxetine in the treatment of attention-deficit/hyperactivity disorder: head-to-head, randomized, double-blind, phase Illb study. CNS Drugs, 27: 1081-92. 


MCQ answers
$1 d \quad 2 d \quad 3 a$

Eli Lilly \& Company (2013) A Study for Assessing Treatment of Patients Ages 10-17 With Bipolar Depression (ClinicalTrials.gov NCT00844857). Accessed 30 Sept 2015

Emslie GJ, Mayes T, Porta G, et al (2010) Treatment of Resistant Depression in Adolescents (TORDIA): week 24 outcomes. American Journal of Psychiatry, 167: 782-91.

Findling RL, McNamara NK, Youngstrom EA, et al (2005) Double-blind 18-month trial of lithium versus divalproex maintenance treatment in pediatric bipolar disorder. Journal of the American Academy of Child and Adolescent Psychiatry, 44: 409-17.

Findling RL, Nyilas M, Forbes RA, et al (2009) Acute treatment of pediatric bipolar I disorder, manic or mixed episode, with aripiprazole: a randomised, double-blind, placebo-controlled study. Journal of Clinical Psychiatry, 70: 1441-51.

Findling RL, Youngstrom EA, McNamara NK, et al (2012) Doubleblind, randomized, placebo-controlled long-term maintenance study of aripiprazole in children with bipolar disorder. Journal of Clinical Psychiatry, 73: 57-63.

Findling RL, Robb A, Bose A (2013a) Escitalopram in the treatment of adolescent depression: a randomized, double-blind, placebo-controlled trial. Journal of Child and Adolescent Psychopharmacology, 23: 468-80.

Findling RL, Correll CU, Nyilas M, et al (2013b) Aripiprazole for the treatment of pediatric bipolar I disorder: a 30-week, randomized, placebo-controlled study. Bipolar Disorders, 15: 138-49.

Findling RL, Kafantaris V, Pavuluri M, et al (2013c) Post-acute effectiveness of lithium in pediatric bipolar I disorder. Journal of Child and Adolescent Psychopharmacology, 23: 80-90.

Findling RL, Pathak S, Earley WR, et al (2014) Efficacy and safety of extended-release quetiapine fumarate in youth with bipolar depression: an 8 week, double-blind, placebo-controlled trial. Journal of Child and Adolescent Psychopharmacology, 24: 325-35.

Franklin ME, Sapyta J, Freeman JB, et al (2011) Cognitive behaviour therapy augmentation of pharmacotherapy in pediatric obsessivecompulsive disorder: the Pediatric OCD Treatment Study II (POTS II) randomized controlled trial. JAMA, 306: 1224-32

Geller B, Luby JL, Joshi P, et al (2012a) A randomised controlled trial of risperidone, lithium, or divalproex sodium for initial treatment of bipolar I disorder, manic or mixed phase, in children and adolescents. Archives of General Psychiatry, 69: 515-28.

Geller DA, March J (2012b) Practice parameter for the assessment and treatment of children and adolescents with obsessive-compulsive disorder. Journal of the American Academy of Child and Adolescent Psychiatry, 51: 98-113.

Gibbons RD, Brown CH, Hur K, et al (2012) Suicidal thoughts and behavior with antidepressant treatment: reanalysis of the randomized placebo-controlled studies of fluoxetine and venlafaxine. Archives of General Psychiatry, 69: 580-87.

Gillies D, Sinn JKH, Lad SS, et al (2012) Polyunsaturated fatty acids (PUFA) for attention deficit hyperactivity disorder (ADHD) in children and adolescents. Cochrane Database of Systematic Reviews, 7: CD007986 (doi:10.1002/14651858.CD007986.pub2).

Goodyer IM, Dubicka B, Wilkinson P, et al (2008) A randomised controlled trial of cognitive behaviour therapy in adolescents with major depression treated by selective serotonin reuptake inhibitors. The ADAPT trial. Health Technology Assessment, 12: iii-iv, ix-60.

Haas M, DelBello MP, Pandina G, et al (2009) Risperidone for the treatment of acute mania in children and adolescents with bipolar disorder: a randomised, double-blind, placebo-controlled study. Bipolar Disorders, 11: 678-700.

Hazell P, Mirzaie M (2013) Tricyclic drugs for depression in children and adolescents. Cochrane Database of Systematic Reviews, 6: CD002317.

Hetrick SE, McKenzie JE, Cox GR, et al (2012) Newer generation antidepressants for depressive disorders in children and adolescents. Cochrane Database of Systematic Reviews, 11: CD004851.
Hughes CW, Emslie GJ, Crismon ML, et al (2007) Texas Children's Medication Algorithm Project: update from Texas Consensus Conference Panel on Medication Treatment of Childhood Major Depressive Disorder. Journal of the American Academy of Child and Adolescent Psychiatry, 46: 667-86.

Ivarsson T, Skarphedinsson G, Kornør H, et al (2015) The place of and evidence for serotonin reuptake inhibitors (SRIs) for obsessive compulsive disorder (OCD) in children and adolescents: views based on a systematic review and meta-analysis. Psychiatry Research, 227: 93-103.

James AC, James G, Cowdrey FA, et al (2015) Cognitive behavioural therapy for anxiety disorders in children and adolescents. Cochrane Database of Systematic Reviews, 2: CD004690.

Kennard BD, Emslie GJ, Mayes TL, et al (2014) Sequential treatment with fluoxetine and relapse-prevention CBT to improve outcomes in pediatric depression. American Journal of Psychiatry, 171: 1083-90.

Kumar A, Datta SS, Wright SD, et al (2013) Atypical antipsychotics for psychosis in adolescents. Cochrane Database of Systematic Reviews, 10: CD009582.

Malhi GS, Geddes JR (2014) Carving bipolarity using a lithium sword. British Journal of Psychiatry, 205: 337-9.

March JS, Vitiello B (2009) Clinical messages from the Treatment for Adolescents With Depression Study (TADS). American Journal of Psychiatry, 166: 1118-23.

Miller M, Swanson SA, Azrael D, et al (2014) Antidepressant dose, age, and the risk of deliberate self-harm. JAMA Internal Medicine, 174: 899-909.

Mohatt J, Bennett SM, Walkup JT (2014) Treatment of separation, generalized, and social anxiety disorders in youths. American Journal of Psychiatry, 171: 741-8.

Noguera A, Ballesta P, Baeza I, et al (2013) Twenty-four months of antipsychotic treatment in children and adolescents with first psychotic episode: discontinuation and tolerability. Journal of Clincial Psychopharmacology, 33: 463-71.

National Institute for Health and Care Excellence (2005a) Depression in Children and Young People: Identification and Management in Primary, Community and Secondary Care (last modified: March 2015) (NICE Clinical Guideline 28). NICE.

National Institute for Health and Care Excellence (2005b) ObsessiveCompulsive Disorder: Core Interventions in the Treatment of ObsessiveCompulsive Disorder and Body Dysmorphic Disorder (NICE Clinical Guideline 31). NICE.

National Institute for Health and Care Excellence (2008) Attention Deficit Hyperactivity Disorder: Diagnosis and Management of ADHD in Children, Young People and Adults (last modified: March 2013) (NICE Clinical Guideline 72). NICE.

National Institute for Health and Care Excellence (2013) Psychosis and Schizophrenia in Children and Young People: Recognition and Management (NICE Clinical Guideline 155). NICE.

National Institute for Health and Care Excellence (2014) Bipolar Disorder: The Assessment and Management of Bipolar Disorder in Adults, Children and Young People in Primary and Secondary Care (NICE Clinical Guideline 185). NICE.

Otasowie J, Castells X, Ehimare UP, et al (2014) Tricyclic antidepressants for attention deficit hyperactivity disorder (ADHD) in children and adolescents. Cochrane Database of Systematic Reviews, 9: CD006997.

Pathak S, Findling RL, Earley WR, et al (2013) Efficacy and safety of quetiapine in children and adolescents with mania associated with bipolar I disorder: a 3-week, double-blind, placebo-controlled trial. Journal of Clinical Psychiatry, 74: e100-9.

Pavaluri MN, Henry DB, Findling RL, et al (2010) Double-blind randomized trial of risperidone versus divalproex in pediatric bipolar disorder. Bipolar Disorders, 12: 593-605. 
Pediatric OCD Treatment Study (POTS) Team (2004) Cognitive-behaviour therapy, sertraline and their combination for children and adolescents with obsessive-compulsive disorder: the Pediatric OCD Treatment Study (POTS) randomized controlled trial. JAMA, 292: 1969-76.

Reeves GM, Keeton C, Correll CU, et al (2013) Improving metabolic parameters of antipsychotic child treatment (IMPACT) study: rationale, design, and methods. Child and Adolescent Psychiatry and Mental Health 7: 31

Ross JS, Tse T, Zarin DA, et al (2012) Publication of NIH funded trials registered in ClinicalTrials.gov: cross sectional analysis. BMJ, 344: d7292.

Royal College of Psychiatrists (2007) Use of Licensed Medicines for Unlicensed Applications in Psychiatric Practice (College Report CR142). Royal College of Psychiatrists.

Ruggiero S, Clavenna A, Reale L, et al (2014) Guanfacine for attention deficit and hyperactivity disorder in paediatrics: a systematic review and meta-analysis. European Neuropsychopharmacology, 24: 1578-90.

Rybakowski JK (2014) Factors associated with lithium efficacy in bipolar disorder. Harvard Review of Psychiatry, 22: 353-7.
Schneider C, Corrigall R, Hayes D, et al (2014) Systematic review of the efficacy and tolerability of clozapine in the treatment of youth with early onset schizophrenia. European Psychiatry, 29: 1-10.

Subcommittee on Attention-Deficit/Hyperactivity Disorder, Steering Committee on Quality Improvement and Management (2011) ADHD: Clinical practice guideline for the diagnosis, evaluation, and treatment of attention-deficit/hyperactivity disorder in children and adolescents. Pediatrics, 128: 1007-22

Taylor E, Dopfner M, Sergeant J, et al (2004) European clinical guidelines for hyperkinetic disorder - first upgrade. European Child and Adolescent Psychiatry, 13 (suppl 1): 7-30.

Tohen M, Kryzhanovskaya L, Calson G, et al (2007) Olanzapine versus placebo in the treatment of adolescents with bipolar mania. American Journal of Psychiatry, 164: 1547-56.

Tramontina S, Zeni PC, Ketzer CR, et al (2009) Aripiprazole in children and adolescents with bipolar disorder comorbid with attention-deficit/ hyperactivity disorder: a pilot randomized clinical trial. Journal of Clinical Psychiatry, 70: 756-64.
MCQs

Select the single best option for each question stem

1 A second-generation antipsychotic is usually chosen to treat psychosis in young people because:

a second-generation antipsychotics have fewer adverse effects than first-generation antipsychotics

b second-generation antipsychotics are more effective than first-generation antipsychotics at treating psychosis

c all second-generation antipsychotics are licensed for use in this age group

d second-generation antipsychotics are better tolerated in the short term

e current guidelines advise avoiding firstgeneration antipsychotics.

2 A 15-year-old girl with OCD who did not respond to initial treatment with sertraline is now taking fluoxetine. The dose was titrated to $60 \mathrm{mg} / \mathrm{day}$, which she has been taking for 3 months, as well as receiving CBT. There has been slight improvement, but she continues to carry out rituals on a daily basis. As a next step the prescriber should consider:

a continuing to prescribe at this dose for another 4 weeks as the full effect from a dose is not seen for at least 16 weeks

b stopping the fluoxetine as it is not licensed to treat OCD in a 15-year-old

c cautiously adding clomipramine to the fluoxetine

d switching to clomipramine because there is evidence of better efficacy

e switching the fluoxetine to a second-generation antipsychotic as this is recommended by NICE.

3 Lisdexamfetamine, a stimulant medicine, has recently been launched in the UK. It:

a may be more effective than atomoxetine

$b$ is contraindicated if there is a risk of misuse or diversion by the patient

c is better tolerated than methylphenidate

d requires administration two or three times a day

e is licensed as a first-line treatment for ADHD.
4 Serotonin syndrome is a potentially life-threatening adverse effect of antidepressants that raise serotonin levels. This risk is increased when:

a antidepressants are used in combination

b SSRIs are used with tramadol

c switching from an SSRI to an SNRI

d augmenting an SSRI with lithium

e all of the above.

5 A 17-year-old girl presents with an acute episode of mania associated with bipolar I disorder. She recently stopped taking risperidone because she didn't like the side-effects. She had gained $3 \mathrm{~kg}$ and was lactating. Suitable alternative treatment options include:

a olanzapine

b valproate

c aripiprazole

d lithium

e all of the above. 